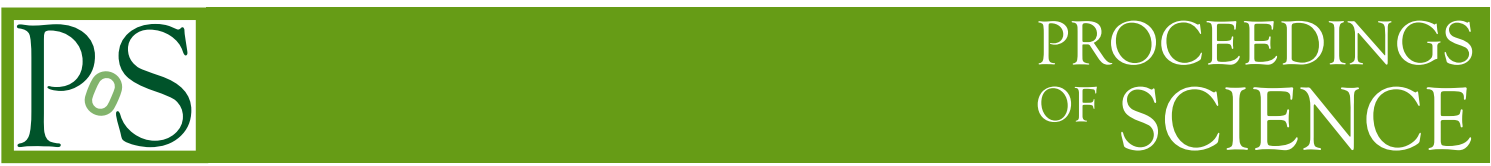

\title{
Gamma-ray selection of DAMPE
}

\section{Zunlei XU ${ }^{* a}$ and Xiang $\mathrm{LI}^{a}$, Jingjing ZANG ${ }^{a}$, Wei JIANG ${ }^{a}$, Yao $\mathrm{LI}^{c}$ on behalf of the DAMPE Collaboration}

${ }^{a}$ Key Laboratory of Dark Matter and Space Astronomy, Purple Mountain Observatory, Chinese Academy of Sciences, Nanjing 210008, China

${ }^{b}$ University of Chinese Academy of Sciences, Beijing, 100012, China

${ }^{c}$ Institute of Modern Physics, CAS

E-mail: खuzIdpmo.ac.cn

Gamma-rays are very important for probing high energy particle accelerators and to study the nature of dark matter. The DArk Matter Particle Explorer (DAMPE), launched on 17th December, 2015, is designed for high energy cosmic-ray and gamma-ray observatory. Gamma-rays constitute only a tiny fraction of all the particles that impinge on our detector, it is thus critical to reject a large amount of ąőbackgroundą́ to get a clean gamma-ray event sample. Here we present an algorithm to identify gamma-rays from the remaining background, using a silicon-tungsten tracker converter which promotes pair conversion and measures the directions of incident particles, and a segmented anti- coincidence detector made from plastic scintillator which measures the charge of particles. We utilize a GEANT4-based Monte Carlo simulation to validate our selection algorith$\mathrm{m}$ and estimate its efficiency. This method has been applied to the flight data, and a preliminary clean GeV gamma-ray sky-map has been obtained.

35th International Cosmic Ray Conference - ICRC2017

10-20 July, 2017

Bexco, Busan, Korea

\footnotetext{
* Speaker.
} 


\section{Introduction}

The DArk Matter Particle Explorer (DAMPE) $[1,2]$ is a general purpose high energy cosmicray and gamma-ray observatory [3, 4, 5], which was successfully launched on December 17th, 2015 and DAMPE presently orbits sun-synchronously at an altitude of $500 \mathrm{~km}$. From top to bottom. DAMPE is composed of four sub-detectors, the Plastic Scintillation Detector (PSD), the SiliconTungsten Tracker (STK), the BGO calorimeter (BGO) and the Neutron Detector (NUD) [1, 2]. PSD is used to measure the charge of incident particles, STK can also measure charges and provide high position resolution tracks simultaneously, and BGO is mainly used to measure the direction and energy of incident particles, and to distinguish electrons and protons.

It is a well established fact that, in the energy range covered by DAMPE's gamma-ray observation[2], the rate of gamma-rays is 3-5 orders of magnitude smaller than that of charged particles. Of these charged particles, more than $99 \%$ are protons and heavy nuclei, and a small fraction are cosmic ray electrons $[6,7,8]$. In order to do scientific analysis of gamma-rays, we thus need to select gammarays from a large number of cosmic rays through offline selection methods. We introduce in this paper the procedure of the gamma-ray selection in DAMPE that features both a high background suppression and a high selection efficiency. The paper is organized as follows: the gamma-ray selection procedure is detailed in Sec 2., including e/p separation using BGO (Sec. 2.1), STK track selection (Sec. 2.2), and charged particle rejection using PSD (Sec. 2.3). With all these, we are able to give a preliminary sky map of DAMPE using the existing flight data (Sec. 3.) We summarize our work in Sec. 4.

\section{Resolving gamma-rays from cosmic rays in DAMPE}

\subsection{Electron/proton separation}

The basic principle of our gamma-ray selection is to reconstruct the incident particle tracks, and then distinguish whether these are gamma-rays or charged particles. To this end we utilize the PSD on the top as anti-coincidence detector, which in the presence of a charged particle shows activity. Due to the limitation of the detection efficiency of the PSD and the reconstructed track angle dispersion[2], there will always some residual charge particles background. Therefore, before using the PSD, this background should be suppressed as much as possible.

The (electromagnetic) showers induced by electrons and gamma-rays in the BGO are very similar, while the shower profile differs when hadrons interact in the BGO. It is therefore possible to use the differences both in the transversal and in the lateral shower profile to remove protons (including other nuclei) first. In DAMPE we utilize both the hardware (including the trigger) to sucessfully suppress the proton background, noting the difference in the e/p energy deposition in the BGO crystals with a general suppression power of $\mathrm{O}(10)$ [2], whereas the remaining protons are filtered using software algorithms. (the suppression power is of the order $\mathrm{O}(10)$ : one involves the detector itself, including the trigger and the different ratios of e/p energy deposition in the BGO crystals

The e/p identification algorithm is inherited from ATIC $[9,10]$. In Fig. 1 we show that using this algorithm as a filter, the total proton suppression power of the method can be several hundreds or even thousand times better than using only the BGO, while retaining electrons and gamma-rays 
at $99 \%$ efficiency with a residual proton background of few percent. Note that we used Monte Carlo simulation data of the detector based on the Geant4 toolkit and a GAUDI-like software framework $[11,12]$.

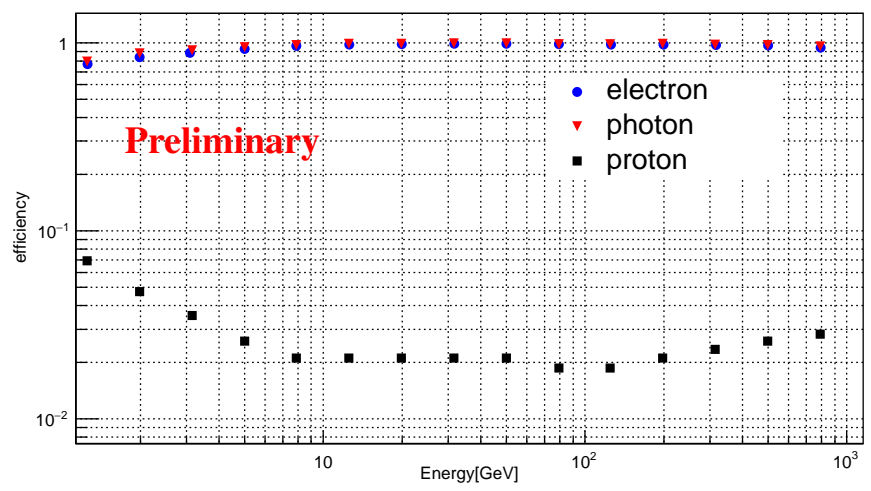

Figure 1: Selection efficiency of proton, electron and gamma-rays after the e/p filter.

\subsection{STK track selection}

In using the PSD as an ACD for gamma-ray identification, a correct track is a prerequisite to locate exactly the PSD strip(s) that are passed by the incident particle.

A single BGO track is reconstructed using an energy centroid method[2]. The standard Kalman filter is then applied to the STK track reconstruction[2]. However, in most cases the backsplash and secondary particles can create tracks in STK. As a result, it is natural to have more than one track reconstructed for a single event, and the average number of tracks will increase with the total energy deposited in DAMPE.

In case that multiple STK tracks are available, we need a variable that quantifies the quality of correctly assigning the true track associated with the incidence particle. Considering several observables, we define an empirical quantity $T Q$ to describe the quality of a track:

$$
T Q=\frac{(1+\text { EnergyRatio })}{\log _{15} \text { Distance }} \times\left[1+\frac{(\text { TrackPoints }-3)}{12}\right],
$$

where "EnergyRatio" is the energy deposition in the STK around the track by $5 \mathrm{~mm}$ divided by the total energy deposited in STK. "Distance" denotes the sum of the distance between the CoGs in the first four BGO layers and the positions when extrapolating the STK track on to the corresponding BGO layers. Finally, "TrackPoints" is the number of points by which the STK track is reconstructed. For the true track, this equation yields the maximum TQ value.

The above defined TQ is validated by applying to Geant 4 simulated electrons, gamma-rays and protons from $1 \mathrm{GeV}$ to $1 \mathrm{TeV}$. We show in Fig. 2 the position error distribution of the STK track, defined as the deviation between the real impact point in a given PSD layer with the predicted impact point as given by the STK track. Given the position error provided by the STK tracks, we use two more neighboring PSD bars on each side of the impacted bar to reject charged particles. 


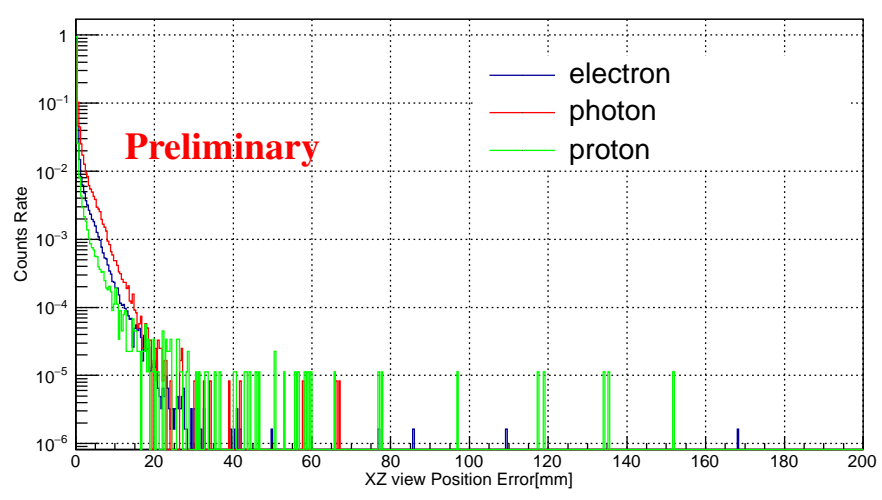

Figure 2: Position error distribution of STK track.

\subsection{Reject charged particles with PSD}

In Fig. 3 we show the largest energy deposition of the PSD bars in X and Y-layers (Edep_x and Edep_y respectively) used for ACD. We can easily identify that the deposited energy of photons is in general smaller than that of electrons. Hence we use the sum of Edep_x and Edep_y as cut (as shown in Fig. 3).

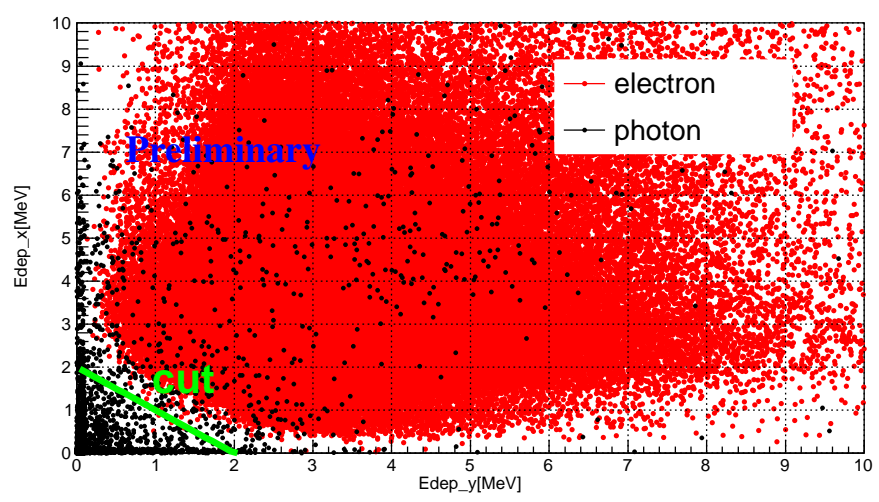

Figure 3: Deposited energy in the PSD (Edep_y vs Edep_x) for both electrons and gamma-rays. The green line indicates the cut made to separate the samples

To determine the optimal value of the threshold applied to the deposited energy in the PSD, we plot in Fig. 4 the residual electron rate as a function of the threshold. It can be seen that the electron residual rate(left black axis) and photon efficiency(right red axis) increases as the threshold increases. In order to balance the rejection efficiency of electrons and the detection efficiency of gamma-rays we use a threshold of $1.5 \mathrm{MeV}$ for low energy events. With this cut value, we achieve a rejection efficiency greater than 0.99999. Since the rejection efficiency of the PSD is higher for high energy electrons and because the energy spectrum deposited in the PSD is softer than that of gamma-rays [13], we choose to increase the above threshold as the energy increases. Given the application of the e/p separation filter along with the PSD threshold filter, we show in Fig. 5 
the acceptance of gama-rays and electrons, based on MC simulations. ${ }^{1}$ Note that after filtering by the selection algorithm the acceptance of electrons is about five orders of magnitude lower than that of gamma-rays. Convolving this acceptance with the spectrum of gamma-rays and electrons as measured by other experiments ${ }^{2}$, we obtain the predicted rate of these particles for DAMPE in Fig. 6. We find that based on these results, that the electron background is about a few percent in the sub-10GeV range, and lower than $1 \%$ at higher energies. Currently, the evaluation of the proton acceptance is hindered due to the lack of sufficient MC data, especially towards the highest energies. However, combining the e/p discrimination and the ACD procedure, we estimate the proton rejection power to be $\sim 10^{7}$.

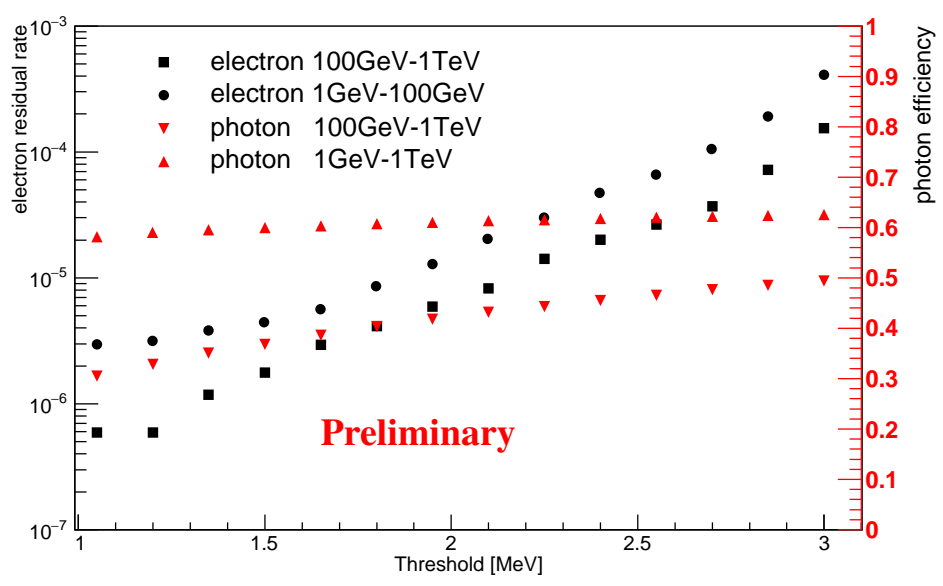

Figure 4: Residual electron rate(left axis) and photon selection efficiency (right axis) versus PSD energy threshold.
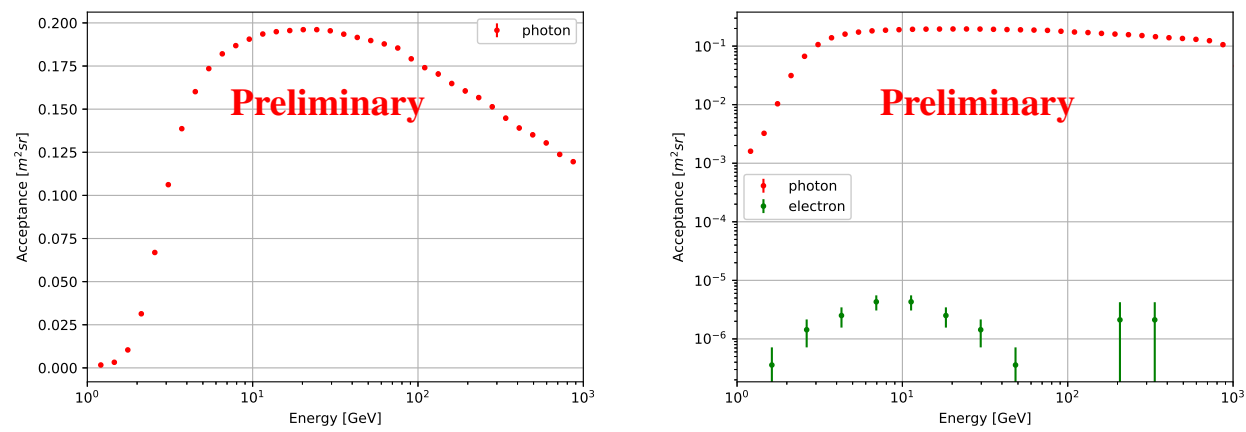

Figure 5: Gamma-ray acceptance in linear scale(left). Gamma-ray and electron acceptances in log scale(right).

\footnotetext{
${ }^{1}$ Here we refer to acceptance as the average effective area times the field of view.

${ }^{2}$ For electrons we use the cosmic ray spectrum as measured by AMS-02 [14] whereas for gamma-rays we assume that the gamma-ray spectrum is given by the isotropic gamma-ray background as measured by Fermi-LAT[15]
} 


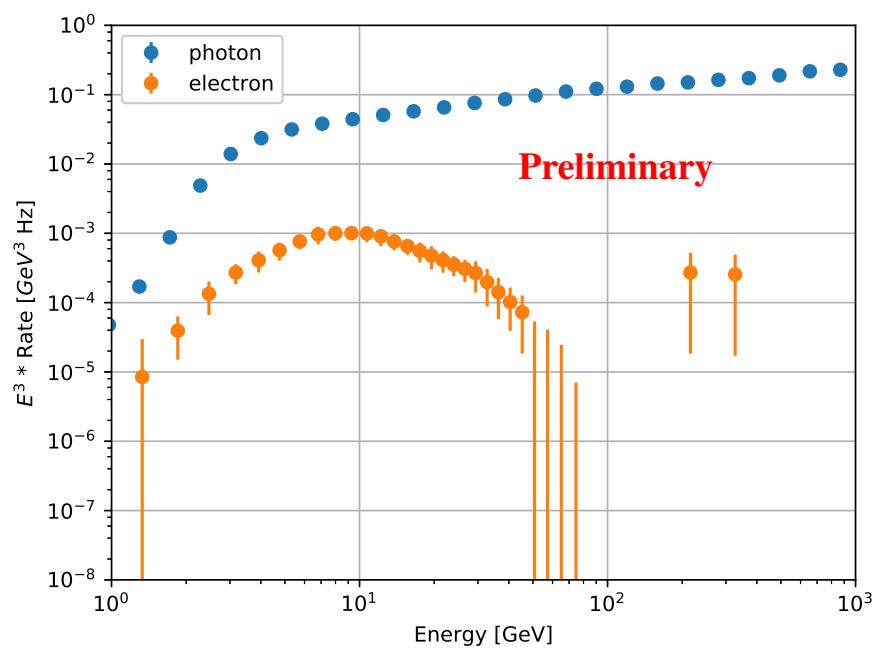

Figure 6: Predicted rate of gamma-rays and contaminated electrons.

\section{Preliminary sky map of DAMPE photon counts}

Applying the gamma-ray selection method to the DAMPE observation that lasts for more than 15 months so far, we are able to produce a gamma-ray sky map, in which various gamma-ray point sources could be identified. Fig. 7 shows the gamma-ray counts map of DAMPE in the energy band from $2 \mathrm{GeV}$ to $1 \mathrm{TeV}$, in which the three brightest sources of the gamma-ray sky, Vela, Geminga and Crab are clearly visible.

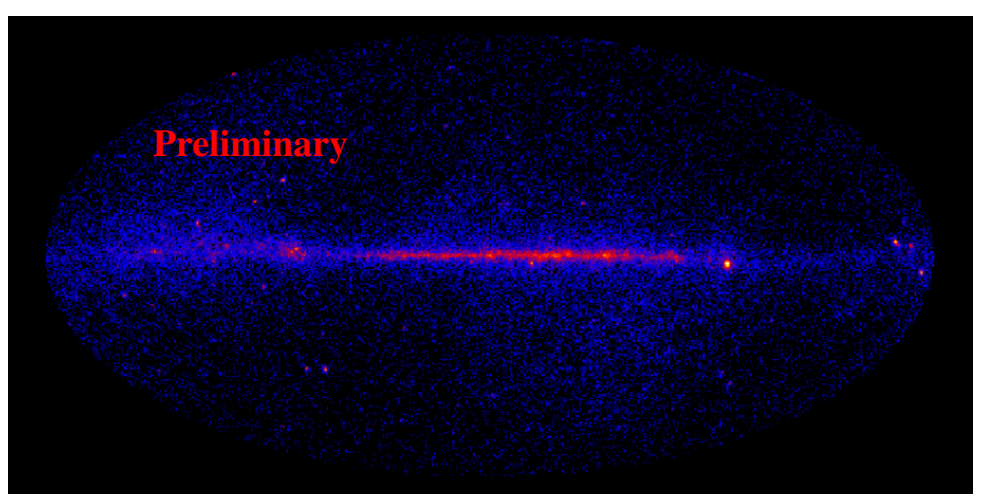

Figure 7: Sky map of DAMPE photon counts. The map is in a Mollweide projection in Galactic coordinates and have energies from $2 \mathrm{GeV}$ to $1 \mathrm{TeV}$ in this work.

\section{Summary}

The particle identification is one of the most important aspects of DAMPE data analysis. We introduce in this paper an algorithm to identify gamma-rays from the bulk of cosmic rays detected by DAMPE. An e/p separation is first applied to exclude the majority of protons based on the 
different shower characteristics of electrons and protons in the BGO calorimeter. A track selecting method is then studied to accurately identify the PSD strip passed by the incident particle. Finally, the charged particles rejection efficiency obtained from Monte Carlo simulation shows that our method has a powerful background suppression level, sufficient to accurately select photons which we demonstrate by showing a map of the gamma-ray sky as seen by DAMPE.

\section{Acknowledgements}

The DAMPE mission is funded by the strategic priority science and technology projects in space science of Chinese Academy of Sciences. The analysis of this work is supported in part by the National Key Research and Development Program of China (No. 2016YFA0400200) and the Strategic Priority Research Program of the CAS "Multi-waveband Gravitational Wave Universe"(Grant No. XDB23040000).

\section{References}

[1] J. Chang, Chin. J. Spac. Sci., 34 (2014) 550.

[2] J. Chang, et al. [DAMPE collaboration] arXiv:1706.08453.

[3] Y. Z. Fan, B. Zhang, \& J. Chang, Int. J. Mod. Phys. D, 19 (2010) 2011.

[4] J. L. Feng, Annu. Rev. Astron. Astrophys. 48, 495 (2010).

[5] Y. F. Liang, et al. Phys. Rev. D, 93 (2016) 103525.

[6] E. S. Seo, et al. Astrophy. J., 378 (1991) 763.

[7] S. W. Barwick, et al. Astrophy. J., 498 (1998) 779.

[8] P. Sreekumar, et al. Astrophy. J., 494 (1998) 523.

[9] Chang, J., et al. Proceedings of the 26th International Cosmic Ray Conference, Salt Lake City, Edited by D. Kieda, M. Salamon, and B. Dingus, Vol.5, 37 (1999).

[10] Chang, J. et al. (The ATIC collaboration). Adv. Spac. Res., 42, 431-436 (2008).

[11] S. Agostinelli, et al. Nucl. Instrum. Methods A, 506 (2003) 250.

[12] C. Wang, et al. arXiv:1604.03219.

[13] Luca Baldini. arXiv:1407.7631.

[14] M. Aguilar et al. (The AMS Collaboration).Phys. Rev. Lett., 113 (2014) 221102.

[15] M. Ackermann et al. [Fermi-LAT Collaboration]. arXiv:1410.3696. 